\title{
Optimization Methods for Energy Consumption Estimation in Wireless Sensor Networks
}

\author{
Mare Srbinovska*1, Marija Cundeva-Blajer ${ }^{2}$ \\ ${ }^{1}$ Department of Electrical Measurement and Materials, Faculty of Electrical Engineering and Information \\ Technologies, Ss Cyril and Methodius University, Rugjer Boskovik 18, PO Box 574, \\ 1000 Skopje, R. Macedonia \\ e-mail: mares@feit.ukim.edu.mk \\ ${ }^{2}$ Department of Electrical Measurement and Materials, Faculty of Electrical Engineering and Information \\ Technologies, Ss Cyril and Methodius University, Rugjer Boskovik 18, PO Box 574, \\ 1000 Skopje, R. Macedonia \\ e-mail: mcundeva@ feit.ukim.edu.mk
}

\section{ABSTRACT}

The main problems in wireless sensor technologies are the constrained energy resources (e. g., battery capacity, processing consumption), and their long-lasting operational capacity in the environment while collecting and sending data to the central station. So, in the design and development of wireless sensor networks, one of the main challenges is to achieve maximal battery life. Real time monitoring by implementation of wireless sensor networks contributes to minimization of potential production risks, emerging mainly from environmental influences and human actions. The main goal in this paper is to obtain minimal energy consumption of wireless sensor nodes while collecting distributed data in environmental parameters monitoring. The communication module and the controller should be in idle state as long as possible when they are not active. Energy consumption changes with the frequency of the transmitted measurement data by the sensors and send/receive configuration of the radio frequency modules. Therefore, all of these parameters should be chosen carefully in order to create an optimal environmental monitoring system. In this contribution the stochastic optimization method-genetic algorithm is used to minimize the energy consumption of the wireless sensor nodes depending on the frequency of the transmitted data and the period of the transmission process. The optimization method is implemented for different scenarios while the frequency of the transmitted data is increasing and the period of transmission of all the active components in a sensor node is increasing.

\section{KEYWORDS}

Wireless sensor networks, Smart sensor energy consumption, Energy optimization, Genetic algorithm, Battery life, Environmental monitoring.

\section{INTRODUCTION}

Wireless Sensor Network (WSN) is composed of sensing nodes that wirelessly transmit data. Sensor nodes are spread in an environment in order to monitor the specific

\footnotetext{
* Corresponding author
} 
physical phenomena such as temperature, humidity, light, pressure. These nodes have many advantages compared with the wired sensor technologies: compact size, mobility feature, ease of installation and low power consumption [1].

Sensor nodes are able to sense the physical parameters, measure the data and gather information from the environment. A typical sensor node is generally composed of power unit, sensor unit, processing unit and communication unit. Sensing and processing unit are responsible for collecting the data and processing the signals acquired from sensors before transferring them to the network. The communication unit task is to transmit the data to the main base station, where it can be further analysed by the user. The power consumption of the supply unit is the main problem in sensor nodes technology because of the constrained energy resources [2]. Two main reasons for the distribution of the energy consumption mainly lead to the asymmetry of the energy consumption between nodes. One is the energy consumption from the communications between nodes and base station, and the other is from the Cluster Head $(\mathrm{CH})$ for receiving data from other nodes [3]. Baggio [4] developed energy-efficient T-Medium Access Control (MAC) protocol in precision agriculture using WSN's. Sensor nodes should work autonomously for a long period of time especially in harsh environment or areas with no available power infrastructure [5]. The limited sensor node energy with the dynamic network topology information has always been the important design concern in WSN's. Clustering of the nodes by grouping the nodes into hierarchies is also an effective way to reduce communication distance and the amount of message [6].

WSN's are used for intelligent monitoring of temperature, humidity, pressure, water level, remote health monitoring of patients, military applications and many other applications. Podgornik et al. [7] present the concept of a platform for analysis of the energy consumption and energy efficiency in urban and industrial areas.

Sensor nodes are usually battery powered wireless devices. The life of the sensor network is mainly influenced by the constrained power resources of the nodes. The small size of the nodes is the main factor for power resource limitations. For example, the total energy that can be stored in a small sensor node is in the range of $1 \mathrm{~J}$ [8]. For typical small systems like Wireless Integrated Network Sensors (WINS) [9], to provide better operation time of the batteries the average current consumption should be less than 30 $\mu \mathrm{A}$. In this type of sensor nodes, the power units are charged from coin cells made from Lithium (Li) (the typical dimensions of the coins are: $2.5 \mathrm{~cm}$ in diameter and $1 \mathrm{~cm}$ in thickness) [9]. One possible solution to extend the duration of the batteries is by energy harvesting [10], which means extracting energy from the environment. One possible solution for energy conservation is by using photovoltaic cells. Self-powered WSN's provide the possibility of very long sensor node life while their deployment would have the least impact on the existing infrastructure. With the integration of renewable energy, especially solar and wind power, it is possible to provide power supply for remote communities. The combination of the renewable energies, wind, solar and energy storage enable sustainable energy production for remote communities and the energy cost can be lower than diesel production as well [11]. Also, Ma et al. [12] made a final decision to take renewable energy, i.e., solar and wind energy or their combination, since it would be the lowest cost option when compared with remote diesel power generation. In addition, they concluded that renewable energy technology is now mature enough to provide utility quality power supply at a reasonable cost.

Therefore, the primary goal in wireless sensor technologies is focused on power conservation while in the traditional networks the main goal is to achieve high Quality of Service (QoS). Actually, the trade-off between energy consumption and QoS in terms of reliable data delivery is active research [13]. It is challenging to optimize the network performances that minimize the energy consumption and in the meanwhile guarantee QoS. 
The minimization of the power node battery consumption is investigated in more detail in order to obtain lower power operation which extends the battery life of the nodes [14].

One of the greatest issues that WSN's faced is energy constraint. It is challenging to prolong energy-power and network life of sensor nodes. Many research topics are focused on efficient energy consumption approaches such as data aggregation, topology construction and energy harvesting in WSN's [15]. Even though there are different methodologies in related works, they have the same ultimate goal which is efficient energy consumption. Therefore, the existing problem of energy constrained resources in WSN's and the possible ways to solve it is the main motive to start our work in this field.

The aim of this study is to use the stochastic method in order to optimize the energy consumption of the wireless sensor nodes which depends on the frequency of the transmitted data and the period of the transmission process.

The paper is organized as follows: in the second section a literature review is given. The third section describes the genetic algorithm implemented in WSN's. The mathematical model and the measurement results for optimization of the energy consumption are presented in the fourth section. Conclusions are presented in the last section.

\section{LITERATURE REVIEW}

In recent years, a diversity of works is related to efficient energy consumption in sensor network technologies. Data aggregation is a technique to eliminate redundant data and reduce energy consumption. In their work, Heinzelman et al. [15] is focused on efficient energy consumption in delivering data from sensor nodes to the base station. Data aggregation protocols eliminate redundant sensor nodes' data sent to the base station. Boonsongsrikul et al. [16] proposed a new topology construction for sensor network topologies in order to minimize bottleneck of data transmission and reception. Most of the works to prolong the life of the sensor nodes are focused on clustering algorithms. One of the most popular Low Energy Adaptive Clustering Hierarchy (LEACH) algorithms for extending the life of the nodes with relatively simple principle and low complexity was proposed by Heinzelman et al. [17].

Several improved algorithms based on LEACH were proposed. Multi-hop LEACH algorithm was proposed by Biradar et al. [18]. In this work, if the Base Station (BS) is out of the scope of the $\mathrm{CH}$, other $\mathrm{CH}$ 's could work as relay nodes for it. Besides the decentralized cluster algorithms, some centralized algorithms were proposed [19], which configured the BS as the main optimizing controller to achieve the selection process of the CH's. To optimize the energy consumption in addition to LEACH, Hybrid Energy Efficient Distributed (HEED) algorithm was also proposed [20]. The HEED clustering protocol adopted the distributed clustering process during which the node with more remaining energy had a higher probability to be the $\mathrm{CH}$. Some authors select the $\mathrm{CH}$ 's based on the residual energy of the sensor nodes [21]. In this work they choose the sensor nodes with the highest residual energy as the $\mathrm{CH}$ nodes, mainly because the $\mathrm{CH}$ consumes more energy than the $\mathrm{nCH}$ nodes. In [22], the authors select the $\mathrm{CH}$ 's based on their proximity to the $\mathrm{BS}$ which is computed in the form of a distance metric. It is assumed that since the transmission distance plays important role in the energy dissipation model, the sensor nodes with the shortest distances to the BS will consume less energy when forwarding packets to the BS.

Some research works are focused on energy harvesting in WSN [23]. Energy harvesting in WSN means gathering energy from the environment or other energy sources and converting it to electrical energy of sensor nodes. Approaches relevant to energy harvesting can be categorized into two groups: based architectures and based protocols. 
In the based architectures, the energy harvesting is related to additional hardware such as solar cells or piezoelectrical materials that convert other sources into electrical energy. Energy storage devices such as recharged batteries or storage capacitors are required for energy-harvesting sensor nodes. When more electrical circuits are added in a sensor node, more energy consumption is required. To prolong its life, rather than using only electrical circuits to measure residual energy, a sensor node should be able to evaluate its remaining energy by learning, reasoning and information shared among multiple nodes. Zhang et al. [23] analysed the maximization of the life in a single cluster topology using the same energy model as LEACH. In the based protocols, an energy harvesting protocol refers to a communication protocol relevant to a sensor node being able to harness energy. Yoo et al. [24] proposed schemes that allow sensor nodes to adjust duty-cycle based on residual energy. Duty - cycle should be adjusted on residual energy in order to reduce the sleep latency and to balance energy consumption.

In this paper we discuss about the possibilities and challenges of our proposed stochastic optimization method-genetic algorithm for effective energy consumption in WSN. We use different scenarios for analysis of the energy consumption of sensor nodes depending on the frequency of the transmitted data and the period of transmission of all the active components. The duty cycles in sensor nodes consist of active and sleep mode. After the initialization of the sensor and transmitting the data to the base station, the sensor node transits to low power mode and stays in this mode until the next transmission period. The transition period between the different modes of operation should be with small duty-cycles in order to obtain maximum efficiency.

Effective energy consumption approaches have different methodologies and performance, but still there are some common aspects such as data delivery from sensor node to the base station, requirements of extra hardware, balancing topologies and scalability in WSN.

\section{RESEARCH METHOD}

The parameters used for the analysis are mainly taken from the previous research in [25]. The duty cycles in active and sleep mode of the energy consuming components in the sensor nodes are measured for different scenarios. In this study the mathematical model for energy consumption optimization in wireless sensor networks is presented. The model is designed according to the different input values which depend on the frequency of the transmitted data and the period of the transmission. For the optimization process the genetic algorithm with population size from 20 to 100 is used.

\section{Description of genetic algorithm implemented in wireless sensor networks}

Genetic Algorithm (GA) is one of the most powerful computational tools. It is very easy to use, but also complex to work with.

This algorithm is originally developed as computer program implemented at the Ss. Cyril and Methodius University in Skopje [26, 27]. At the beginning, the GA begins with the primary units (chromosomes) stochastic generation. It is important to note that each of the chromosomes consists of the same number of genes.

The population size can vary from 20 to 100. In every repetition step in the GA the period of estimation, selection and reproduction of the population repeats. So, the possible optimal solution can be represented by each of the unit. The following criterion represents one possible solution for each unit: for goal function value and each solution is the value of the optimal goal function and on each solution is set an appropriate measure. With the comparison of all appropriate measures, a decision can be made as to which of the units can make the next generation and what is the probability in the next step of selection. 
Mathematical model for energy consumption optimization in wireless sensor networks

The life of the sensor nodes is directly influenced by the energy consumption of the components built in the modules [25]. From all the components integrated in the sensor nodes, the transmitting module is the most energy consuming part. Generally, the energy consumption can be the result of four modes of operation:

- The sleeping mode, where the microprocessor and the radio are not operating or the state is called idle mode. This state lasts until some external events which is the reason to enter in;

- Processing mode, when the sensor transits from the processing to:

○ Transmitting;

- Or receiving mode, the energy consumption can be increased for $50 \%$ of the energy consumption in the processing mode.

In order to obtain maximum efficiency in the operation modes, one possible solution is to transit between the modes in a small duty cycles [28]. The energy consumption between two nodes that are transmitting data information can be written as:

$$
A=A_{\text {sensor }}+A_{\text {mc }}+A_{\text {transmitter }}
$$

The total energy consumed for transmission of one complete information package $(A)$ is result of the energy consumption of each component: the sensor $\left(A_{\text {sensor }}\right)$, microcontroller $\left(A_{\mathrm{mc}}\right)$ and radio transmitter $\left(A_{\text {transmitter }}\right)$.

When the number of the sensor nodes grows in the network, it is necessary to estimate the power supply of the whole system in order to obtain appropriate operation of the network. The energy $A$ that is dissipated in each node can be calculated as:

$$
\begin{gathered}
A=P \times t \\
A=V_{\mathrm{dd}} \times I \times t
\end{gathered}
$$

where $V_{\mathrm{dd}}=3.3 \mathrm{~V}$ indicates the power supply voltage, $I$ represents the current consumption in different modes of operation and $t$ denotes the time for sending data to the sensor node.

The total energy consumption of the sensor nodes can be calculated mainly as a result of the consumption of the three main components in the sensor module: the sensor, microcontroller and the radio module. The current consumption of each component also depends on the modes of operation. For example, when the sensor is in the sleep mode, the current consumption is $0.6 \mu \mathrm{A}$, but in active mode the consumption is $0.9 \mu \mathrm{A}$.

The current consumption of the microcontroller can vary from $0.9 \mu \mathrm{A}$ when it is in low power mode to $2.7 \mathrm{~mA}$ when it is in active mode.

These differences in the energy consumption modes of operation is especially clear in the transmission mode, when the transceiver is in sleep mode, the current consumption is only $0.4 \mu \mathrm{A}$, but in transmitting mode the current consumption can increase to $21.2 \mathrm{~mA}$.

In order to calculate the energy consumption of all sensor node components different modes of operation (time to initialize the sensor), active and sleep mode and transmitting mode should be included. The equation related to the energy consumption of the sensor is:

$$
A_{\text {sensor }}=P_{\text {sensorst }} \times t_{\text {start }}+P_{\text {sensorsl }} \times t_{\mathrm{sl}}+P_{\text {measure }} \times t_{\mathrm{tr}}+P_{\mathrm{tx}} \times t_{\mathrm{tx}}
$$

where $P_{\text {sensorsl }}$ is the power consumption of the sensor module in the initialization period, $t_{\text {start }}$ is the time to initialize the sensor, $P_{\text {sensorsl }}$ is the power consumption in sleep mode, 
$t_{\mathrm{sl}}$ is the time when the sensor is in sleep mode, $P_{\text {measure }}$ is the power consumption when the sensor is starting to transmit the data, $t_{\text {tr }}$ is the time for the sensor to prepare for the transmission period, $P_{\mathrm{tx}}$ is the power consumption in the transmission mode and $t_{\mathrm{tx}}$ is the transmission period.

In Table 1 these time values are defined:

Table 1. Values for the time intervals

\begin{tabular}{cccc}
\hline \multicolumn{5}{c}{ Time } \\
\hline$t_{\mathrm{start}}[\mathrm{ms}]$ & $t_{\mathrm{s} 1}[\mathrm{~ms}]$ & $t_{\mathrm{tr}}[\mathrm{ms}]$ & $t_{\mathrm{tx}}[\mathrm{ms}]$ \\
\hline 11 & $10-120$ & 11 & $40-120$ \\
\hline
\end{tabular}

The energy consumption $\left(A_{\mathrm{mc}}\right)$ of the microcontroller can be calculated as:

$$
A_{\mathrm{mc}}=P_{\mathrm{mclow}} \times\left(t_{\mathrm{start}}+t_{\mathrm{sl}}\right)+P_{\mathrm{mchigh}} \times t_{\mathrm{tx}}
$$

where $P_{\text {mclow }}$ is the power consumption of the microcontroller in low power mode and $P_{\text {mchigh }}$ is the power consumption when the microcontroller is in active mode.

The energy consumption of the radio frequency module in the transmitting mode $A_{\text {transmitter }}$ can be calculated according to the following formula:

$$
A_{\text {transmitter }}=P_{\mathrm{S}} \times\left(t_{\mathrm{start}}+t_{\mathrm{sl}}\right)+P_{\mathrm{tx}} \times t_{\mathrm{tx}}
$$

where $P_{\mathrm{S}}$ is the power consumption of the radio frequency module in sleep mode, and $P_{\mathrm{tx}}$ is the power consumption in the transmitting mode.

In order to obtain optimization in the energy consumption of the sensor node, the length of the data packet information should be included. The time for transmitting the data can be calculated by:

$$
t_{\mathrm{tx}}=\frac{t_{\mathrm{txn}}}{n}
$$

where $t_{\mathrm{tx}}$ is the time for sending single information packet.

$$
t_{\mathrm{txn}}=N_{\mathrm{P}} \frac{8}{0.001}
$$

where $t_{\mathrm{txn}}[\mathrm{ms}]$ is the total transmitting time depending on the length of the packet information.

$$
N_{\mathrm{P}}=N_{\mathrm{mac}}+N_{\mathrm{com}}+N_{\mathrm{IP}}+N_{\text {data }}
$$

where $N_{\mathrm{P}}$ is the total information package length including the information package MAC heading length $\left(N_{\mathrm{mac}}\right)$, the common header $\left(N_{\mathrm{com}}\right)$, IP header $\left(N_{\mathrm{IP}}\right)$ and the data string length in the information package $\left(N_{\text {data }}\right)$.

\section{RESULTS AND DISCUSSION}

For the optimization process the input values are chosen to be data packets with different transmission period and different data package length as the energy consumption mainly depends from these parameters.

The input parameters in the calculation of the energy consumption using the GA optimisation process are given in Table 2. 
Table 2. Input variables for GA with the mapping range

\begin{tabular}{ccc}
\hline Input variables & Minimum & Maximum \\
\hline$X[1]=N_{\text {mac }}[$ bytes $]$ & 2 & 100 \\
$X[2]=N_{\text {data }}[$ bytes $]$ & 6 & 32 \\
$X[3]=n$ & 1 & 10 \\
\hline
\end{tabular}

The optimization goal function is defined according to the following equation of the total energy $A$ consumed for transmission of one complete information package:

$$
A=n\left\{\begin{array}{l}
\left(P_{\text {sensorst }} \times t_{\text {start }}+P_{\text {sensorsl }} \times t_{\mathrm{sl}}+P_{\text {measure }} \times t_{\mathrm{tr}}+P_{\mathrm{tx}} \times t_{\mathrm{tx}}\right)+ \\
{\left[P_{\mathrm{mclow}} \times\left(t_{\mathrm{start}}+t_{\mathrm{sl}}\right)+P_{\mathrm{mchigh}} \times t_{\mathrm{tx}}\right]+} \\
{\left[P_{\mathrm{S}} \times\left(t_{\mathrm{start}}+t_{\mathrm{sl}}\right)+P_{\mathrm{tx}} \times t_{\mathrm{tx}}\right]}
\end{array}\right\}
$$

The purpose of the optimization process is to achieve minimum consumed energy per information package. However, the original GA program is used to optimize the goal function and in the optimization process the auxiliary goal function $\left(f_{\mathrm{opt}}\right)$ is given by the following formula:

$$
\begin{gathered}
f_{\text {opt } 1}=\frac{1}{A_{\text {sensor }}+1} \\
f_{\text {opt } 2}=\frac{1}{A_{\mathrm{mc}}+1} \\
f_{\text {opt } 3}=\frac{1}{A_{\text {transmitter }}+1} \\
f_{\text {opt }}=f_{\text {opt } 1}+f_{\text {opt } 2}+f_{\text {opt3 }}
\end{gathered}
$$

The genetic parameters to obtain optimal solution are calculated according to the following values: cross-over probability is 0.65 , mutation probability 0.03 , population size 16, maximal number of generations 30,000.

In Figures 1-4, the GA optimisation process is presented through the 30,000 generations. The main purpose to optimize the energy consumption is to minimize the parameters $N_{\mathrm{P}}, N_{\text {data }}, N_{\mathrm{mac}}$ as well as optimization of the total transmission time per information package of the sensor node.

In Figures 5-8, the optimal output values for the total information package length, total transmission time per information package and the total energy consumption of the sensor node components are presented.

The optimal value for the energy consumption of the sensor node, $A=780.0901 \mu \mathrm{J}$ is obtained for the following optimal input variables presented in Table 3.

From the Table 3 we can conclude that in the optimization process the length of the MAC heading information package is equivalent to the minimal value. The total information package data is equivalent to data string length in the information package. The optimal energy consumption of the sensor node per information package is presented on Figure 9. 
Goal function

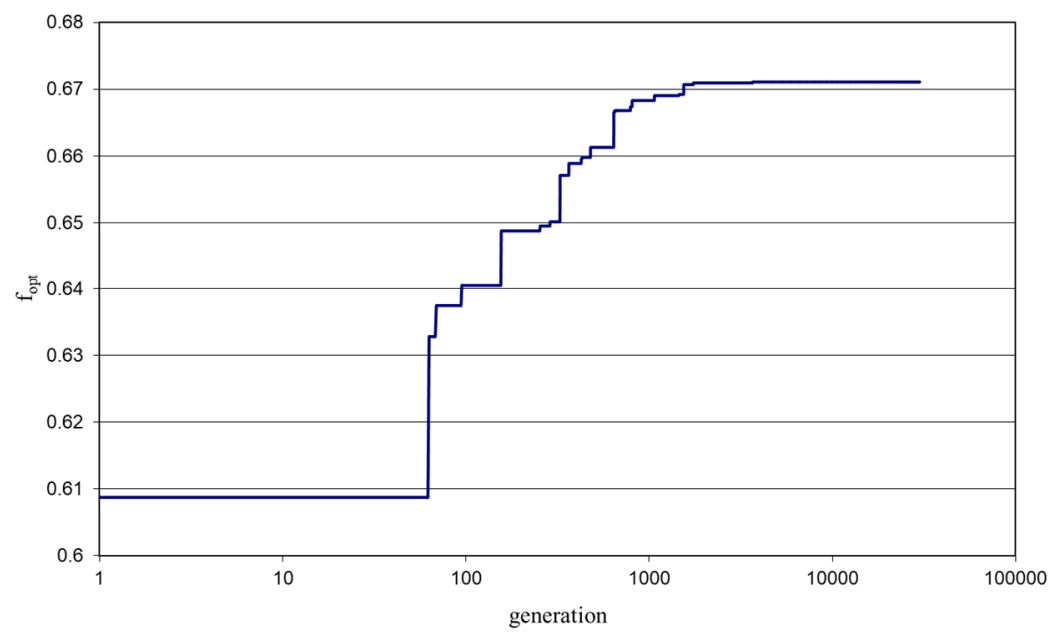

Figure 1. Optimal goal function

Optimization input variable 1

Information package MAC heading length (transmitting mode)

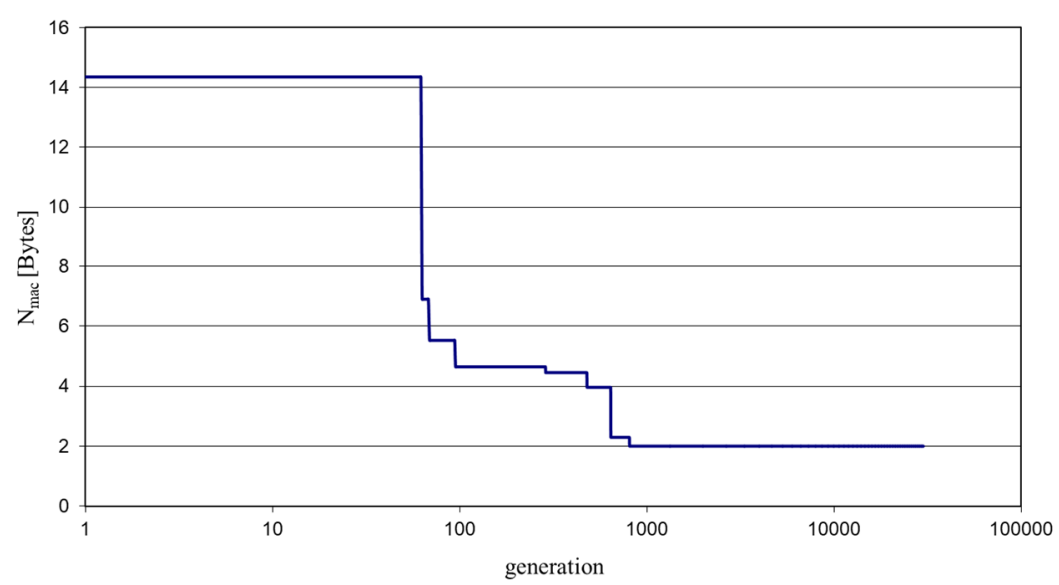

Figure 2. Optimization of the input variable 1 (information package MAC heading length)

Optimization input variable 2

Data string length in the information package (transmitting mode)

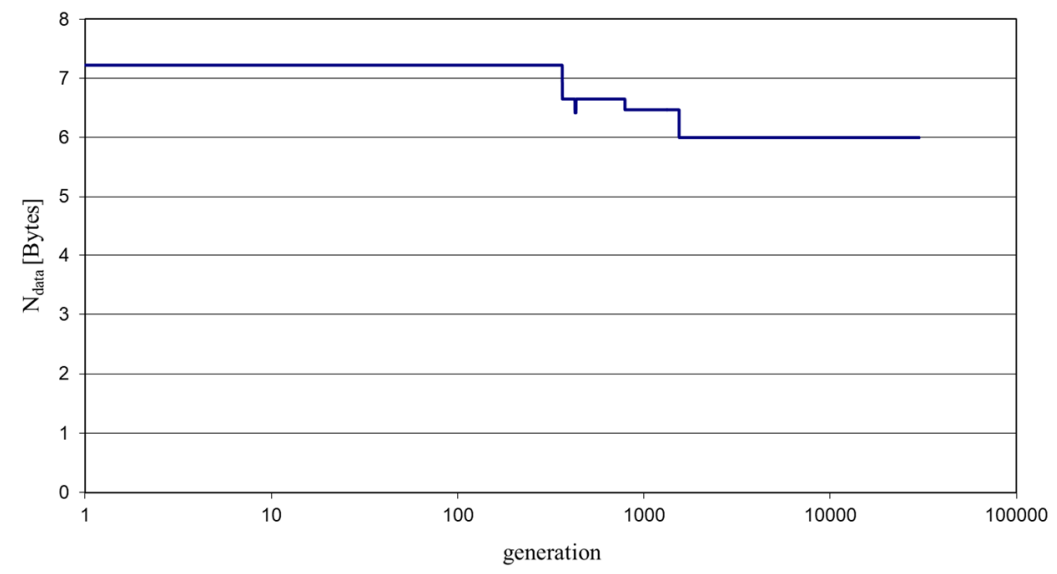

Figure 3. Optimization of the input variable 2 (data string length in the information package) 
Optimization input variable 3

Number of data strings per information package (transmitting mode)

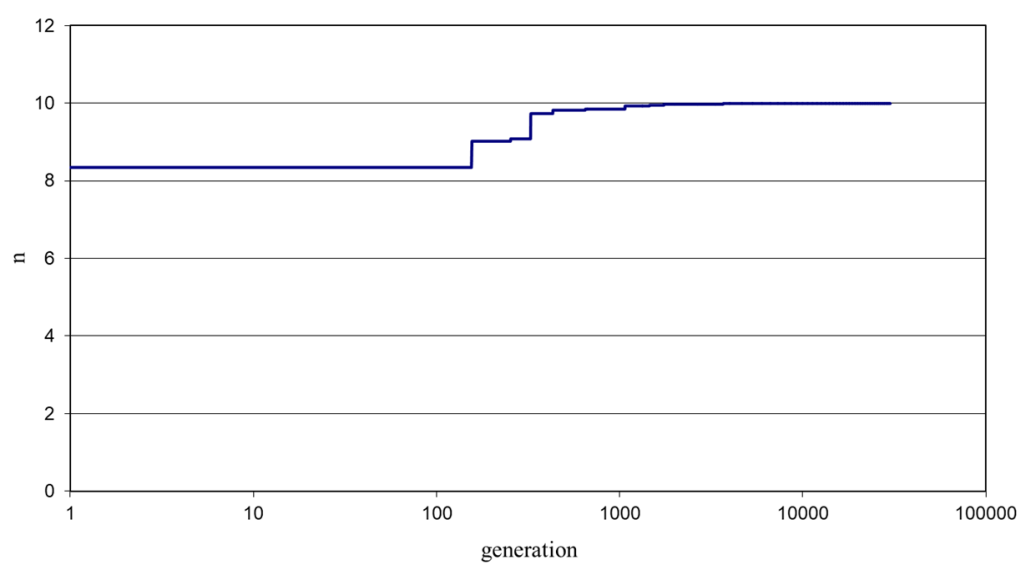

Figure 4. Optimization of input variable 3 (number of data strings per information package of the sensor node in transmitting mode)

Total information package length

(transmitting mode)

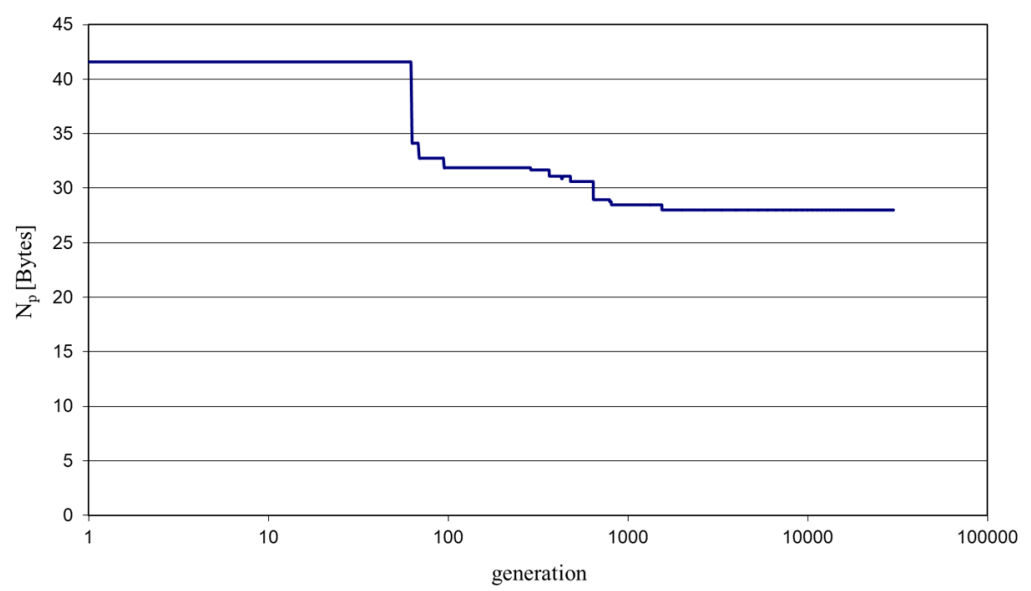

Figure 5. Total information package length (transmitting mode)

Total energy consumption per information package of the sensor (transmitting mode)

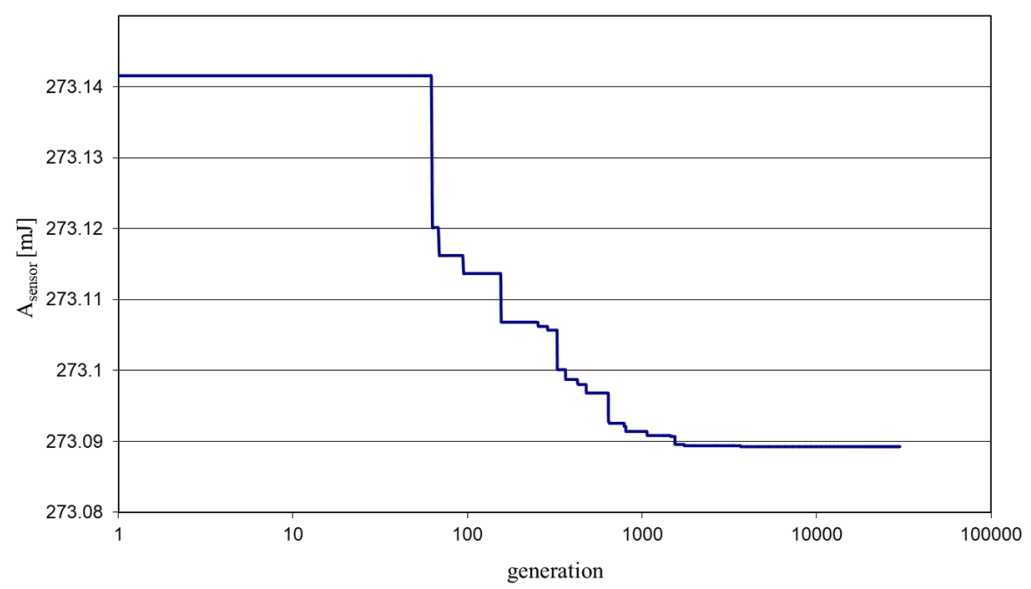

Figure 6. Total transmission time per information package of the sensor node (transmitting mode) 
Total energy consumption per information package of the microcontroller

(transmitting mode)

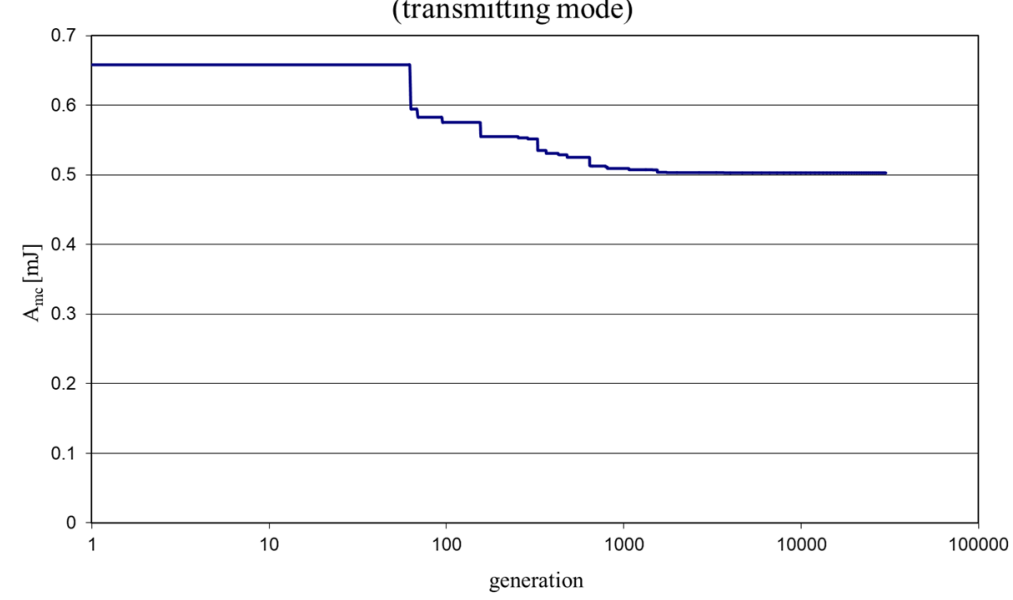

Figure 7. Total energy consumption per information package of the microcontroller

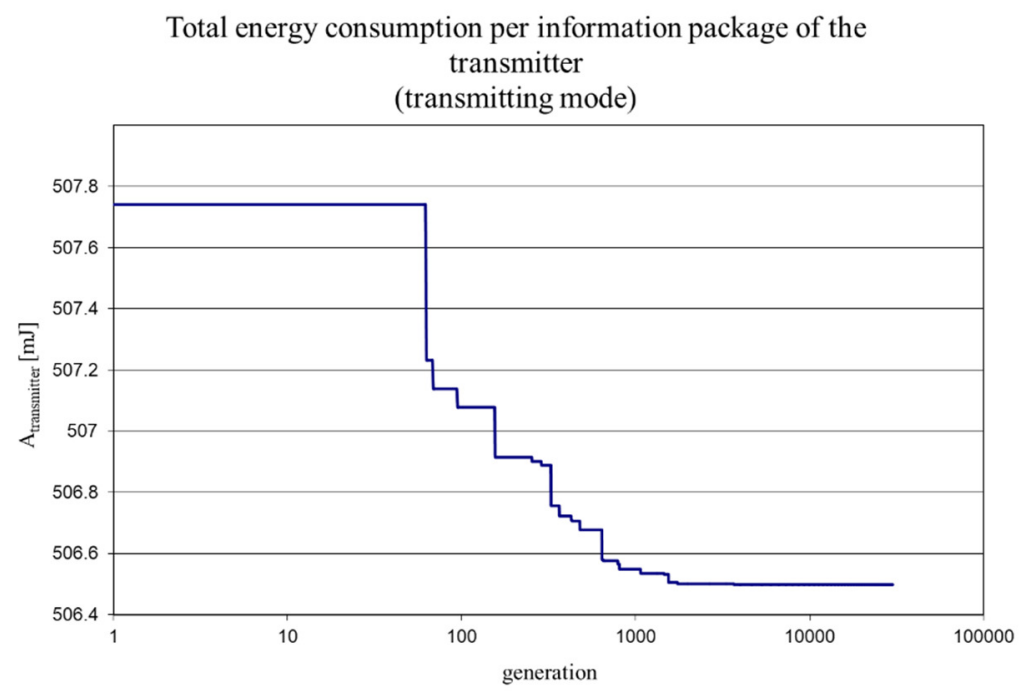

Figure 8. Total energy consumption per information package of the transmitter

Table 3. Optimal input variables for the optimization process

\begin{tabular}{cc}
\hline Input variables & Optimal \\
\hline$X[1]=N_{\text {mac }}[$ bytes $]$ & 2 \\
$X[2]=N_{\text {data }}[$ bytes $]$ & 6 \\
$X[3]=n$ & 10 \\
$f_{\text {opt }}$ & 0.671086 \\
\hline
\end{tabular}

In Table 4 some of the optimal results (total energy consumption of the sensor module, total energy consumption of the microcontroller, total energy consumption of the transmitter, total transmission time per information package of the sensor node and total information package length) are shown.

The purpose of the optimization process is to achieve minimum consumed energy of the sensor nodes per information package. However, the original GA program maximizes the goalfunction and it can be concluded that to obtain minimal energy consumption the total information package length of the data should be bigger and the frequency of the transmitted package information should be sent in long time intervals. The total transmission time per information package of the sensor node is $0.224 \mathrm{~ms}$. 
Total energy consumption per information package of the sensor node (transmitting mode)

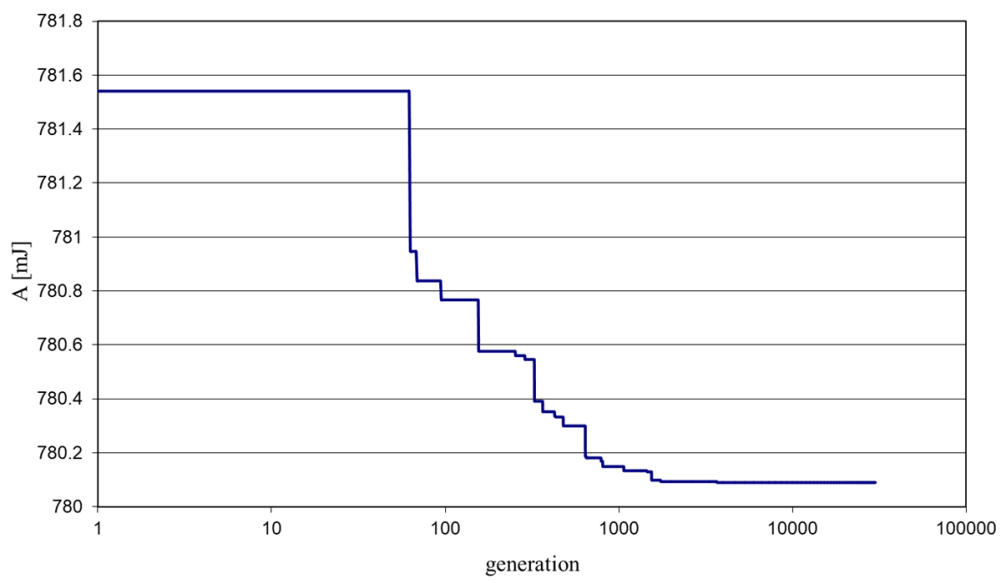

Figure 9. Total energy consumption per information package of the sensor node

Table 4. Results for output variables (optimal values)

\begin{tabular}{cc}
\hline Output variables & Optimal values \\
\hline Total energy consumption per sensor node $(A)$ & $780.0901 \mu \mathrm{J}$ \\
Total energy consumption of the sensor module $\left(A_{\text {sensor }}\right)$ & $273.0893 \mu \mathrm{J}$ \\
Total energy consumption of the microcontroller $\left(A_{\mathrm{mc}}\right)$ & $0.502704 \mu \mathrm{J}$ \\
Total energy consumption of the transmitter $\left(A_{\text {transmitter }}\right)$ & $506.498 \mu \mathrm{J}$ \\
Total information package length $\left(N_{\mathrm{P}}=N_{\text {data }}\right)$ & $0.224 \mathrm{~ms}$ \\
& $28 \mathrm{~B}$ \\
\hline
\end{tabular}

\section{CONCLUSIONS}

In this paper, to minimize the energy consumption of the wireless sensor nodes the stochastic method is used-genetic algorithm that is optimized depending on the frequency of the transmitted data and the period of the transmission process of the sensor nodes. From the results of the optimization process the optimal input parameters for minimal energy consumption of the sensor node are derived.

In the optimization process the energy consumption of the sensor nodes is calculated as a result of all the components consumers in the sensor node: sensor module, microcontroller and the radio transmitter. Minimization of the energy consumption of sensor nodes depending on the frequency of the transmitted data and the period of transmission of all the components are analysed depending on different duty cycles in sensor nodes (active and sleep mode).

By using the GA the minimum consumed energy per information package is calculated. It can be concluded that the frequency of the transmitted data packets should be sent in time intervals with bigger information packages in order to obtain minimal energy consumption.

For further investigation the number of optimization input variables could be increased. The applied methodology is universal and can be applied to other WSN optimization problems.

\section{NOMENCLATURE}

$A$

total energy consumption of the sensor node

$A_{\mathrm{mc}} \quad$ total energy consumption of the microcontroller 


\begin{tabular}{|c|c|}
\hline$A_{\text {transmitter }}$ & total energy consumption of the transmitter \\
\hline & current consumption of the sensor node \\
\hline$n$ & number of data strings \\
\hline$N_{\text {data }}$ & data string length in the information package \\
\hline$N_{\text {IP }}$ & IP header $\left(N_{\mathrm{IP}}\right)$ data string length in the information package \\
\hline$N_{\text {mac }}$ & information package MAC heading length \\
\hline$N_{\mathrm{P}}$ & total information package length \\
\hline$P$ & power consumption of the sensor node \\
\hline$P_{\text {mchigh }}$ & power consumption when the microcontroller is in active mode \\
\hline$P_{\text {mclow }}$ & power consumption of the microcontroller in low power mode \\
\hline$P_{\text {measure }}$ & $\begin{array}{l}\text { power consumption when the sensor is starting to transmit } \\
\text { the data }\end{array}$ \\
\hline$P_{\mathrm{S}}$ & power consumption of the radio frequency module in sleep mode \\
\hline$P_{\text {sensorsl }}$ & power consumption in sleep mode \\
\hline$P_{\text {sensorst }}$ & $\begin{array}{l}\text { power consumption of the sensor module in the initialization } \\
\text { period }\end{array}$ \\
\hline$P_{\mathrm{tx}}$ & power consumption in the transmission mode \\
\hline$t$ & time for sending data to the sensor node \\
\hline$t_{\mathrm{s} 1}$ & time when the sensor is in sleep mode \\
\hline$t_{\text {start }}$ & time to initialize the sensor \\
\hline$t_{\mathrm{tr}}$ & time for the sensor to prepare for the transmission period \\
\hline$t_{\mathrm{tx}}$ & $\begin{array}{l}\text { time for sending single information packet } \\
\text { (transmission period) }\end{array}$ \\
\hline$t_{\mathrm{txn}}$ & $\begin{array}{l}\text { total transmitting time depending on the length of the } \\
\text { packet information }\end{array}$ \\
\hline$V_{\mathrm{dd}}$ & power supply voltage \\
\hline
\end{tabular}

$[\mu \mathrm{J}]$

$[\mathrm{mA}]$

[-]

[bytes]

[bytes]

[bytes]

[bytes]

[mW]

$[\mathrm{mW}]$

$[\mathrm{mW}]$

$[\mathrm{mW}]$

$[\mathrm{mW}]$

$[\mathrm{mW}]$

$[\mathrm{mW}]$

$[\mathrm{mW}]$

[ms]

$[\mathrm{ms}]$

[ms]

[ms]

$[\mathrm{ms}]$

$[\mathrm{ms}]$

$[\mathrm{V}]$

\section{REFERENCES}

1. Akyildiz, I., Su, W., Sankarasubramaniam, Y. and Cayirci, E., Wireless Sensor Networks: A Survey, IEEE Communications Magazine, Vol. 40, No. 8, pp 102-114, 2002, https://doi.org/10.1109/MCOM.2002.1024422

2. Keshtgary, M. and Deljoo, A., An Efficient Wireless Sensor Network for Precision Agriculture, Canadian Journal on Multimedia and Wireless Networks, Vol. 3, No. 1, pp 1-5, 2012.

3. Anastasi, G., Conti, M., Di Francesco, M. and Passarella, A., Energy Conservation in Wireless Sensor Networks, A Survey, Ad Hoc Networks, Vol. 7, No. 3, pp 537-568, 2009, https://doi.org/10.1016/j.adhoc.2008.06.003

4. Baggio, A., Wireless Sensor Networks in Precision Agriculture (Report), ACM Workshop on Real World Wireless Sensor Networks (REALWSN), Delft University of Technology, Delft, The Netherland, 2005.

5. Nasso, Di N., Boscoa, S., Di Bene, C., Coli, A., Mazzoncini, M. and Bonari, E., Energy Efficiency in Long-term Mediterranean Cropping System with Different Management Intensities, Energy, Vol. 36, No. 4, pp 1924-1930, 2011, https://doi.org/10.1016/j.energy.2010.06.026

6. Estrin, D., Govindan, R., Heidemann, J. and Kumar, S., Next Century Challenges: Scalable Coordination in Sensor Networks, Proceedings of ACM MobiCom '99, pp 263-270, Washington, USA, 1999, https://doi.org/10.1145/313451.313556

7. Podgornik, A., Sucic, B. and Urosevic, Lj., The Concept of an Interactive Platform for Real Time Energy Consumption Analysis in a Complex Urban Environment, Journal of Sustainable Development of Energy, Water and Environment Systems, Vol. 3, No. 1, pp 79-94, 2015, https://doi.org/10.13044/j.sdewes.2015.03.0006 
8. Porret, A., Melly, T., Enz, C. C. and Vittoz, E. A., A Low-power Low-voltage Transceiver Architecture Suitable for Wireless Distributed Sensors Network, Proceedings of the IEEE International Symposium on Circuits and Systems'00, Geneva, Switzerland, pp 56-59, 2000, https://doi.org/10.1109/ISCAS.2000.857025

9. Vardhan, S., Wilczynski, M., Pottie, G. and Kaiser, W. J., Wireless Integrated Network Sensors (WINS): Distributed In Situ Sensing for Mission and Flight Systems, Proceedings of the IEEE Aerospace Conference, Vol. 7, pp 459-463, Big Sky, USA, 2000, https://doi.org/10.1109/AERO.2000.879313

10. Rabaey, J. M., Ammer, M. J., Silva, J. L., Patel, D. and Roundy, S., PicoRadio Supports Ad Hoc Ultra-low Power, IEEE Computer Magazine, Vol. 33, No. 7, pp 42-48, 2000, https://doi.org/10.1109/2.869369

11. Fedak, W., Anweiler, S., Ulbrich, R. and Jarosz, B., The Concept of Autonomous Power Supply System Fed with Renewable Energy Sources, Journal of Sustainable Development of Energy, Water and Environment Systems, Vol. 5, No. 4, pp 579-589, 2017, https://doi.org/10.13044/j.sdewes.d5.0160

12. Ma, T., Yang, H., Lu, L. and Peng, J., Optimal Design of an Autonomous Solar-windpumped Storage Power Supply System, Applied Energy, Vol. 160, pp 728-736, 2015, https://doi.org/10.1016/j.apenergy.2014.11.026

13. Mancuso, M. and Bustaffa, F., A Wireless Sensors Network for Monitoring Environmental Variables in a Tomato Greenhouse, Proceedings of the IEEE International Workshop on Factory Communication Systems, Torino, Italy, June 27-30, pp 107-110, 2006, https://doi.org/10.1109/WFCS.2006.1704135

14. Srbinovska, M., Gavrovski, C., Dimcev, V., Krkoleva, A. and Borozan, V., Environmental Parameters Monitoring in Precision Agriculture using Wireless Sensor Networks, Journal of Cleaner Production, Vol. 88, pp 297-307, 2015, https://doi.org/10.1016/j.jclepro.2014.04.036

15. Heinzelman, W. R., Chanrakasan, A. and Balakrishnan, H., An Application-Specific Protocol Architecture for Wireless Microsensor, Transactions of the IEEE, Wireless Communications, Vol. 1, No. 4, pp 660-670, 2002, https://doi.org/10.1109/TWC.2002.804190

16. Boonsongsrikul, A., Park, S. K. and Shin, S. H. An Adaptively Balancing Workload Protocol on Query Trees for Maximizing Life in Sensor Networks, Proceedings of the $12^{\text {th }}$ International Conference on Advanced Communication Technology (ICACT2012), pp 195-200, Paris, France, 2012.

17. Heinzelman, W. R., Chandrakasan, A. and Balakrishnan, H., Energy Efficient Communication Protocol for Wireless Microsensor Networks, Proceedings of Hawaii International Conference on System Sciences, pp 3005-3014, Washington, D. C., USA, 2000, https://doi.org/10.1109/HICSS.2000.926982

18. Biradar, R, V., Sawant, R., Mudholkar, R. R. and Patil, V. C., Inter-intra Cluster Multihop-leach Routing in Self-organizing Wireless Sensor Networks, International Journal of Research and Reviews in Computer Science, Vol. 2, No. 1, pp 88-95, 2011.

19. Quynh, T. N., Phung, K.-H. and Quo, H. V., Improvement of Energy Consumption and Load Balance for Leach in Wireless Sensors Networks, Proceedings of International Conference on ICT Convergence (ICTC), pp 1525-1528, Jeju Island, South Korea, 2012, https://doi.org/10.1109/ICTC.2012.6387206

20. Younis, O., Fahmy, S., A Hybrid, Energy-efficient, Distributed Clustering Approach for Ad Hoc Sensor Networks, Transaction on IEEE Mobile Computing, Vol. 3, No. 4, pp 366-379, 2004, https://doi.org/10.1109/TMC.2004.41

21. Lin, D. and Wang, W., A Game Theory Based Energy Efficient Clustering Routing protocol for WSNs, Journal of Wireless Networks, Vol. 23, No. 4, pp 1101-1111, 2017, https://doi.org/10.1007/s11276-016-1206-2 
22. Lee, S., Yoo, J. and Chung, T., Distance-based Energy Efficient Clustering for Wireless Sensor Networks, Proceedings of the $29^{\text {th }}$ Annual IEEE International Conference on Local Computer Networks, Tampa, FL, USA, 16-18 November, 2004.

23. Zhang, P., Xiao, G. and Tan, H. P., A Preliminary Study on Life Maximization in Clustered Wireless Sensor Networks with Energy Harvesting nodes, Proceedings of the $8^{\text {th }}$ International Conference on Information, Communications \& Signal Processing (ICICS), pp 1-5, 2011.

24. Yoo, H., Shim, M. and Kim, D., Dynamic Duty-Cycle Scheduling Schemes for EnergyHarvesting Wireless Sensor Networks, IEEE Communication Letters, Vol. 16, No. 2, pp 202-204, 2012, https://doi.org/10.1109/LCOMM.2011.120211.111501

25. Srbinovska, M., Dimitrov, D., Gavrovski, C. and Dimcev, V., Loss of Load Probability of Wireless Sensor Networks Powered by Photovoltaic Cells, Journal of Environmental Protection and Ecology, Vol. 17, No. 3, pp 1058-1065, 2016.

26. Cundeva-Blajer, M., Optimization Methods in Metrology of Electrical Quantities, Proceedings of the $21^{\text {st }}$ IMEKO World Congress, Prague, Czech Republic, Aug. 30-Sept. 4, pp 1903-1908, 2015.

27. Cvetkovski, G., Petkovska, L. and Gair, S., Specific Power as Objective Function in GA Optimal Design of Permanent Magnet Disc Motor, COMPEL: Int. Jour. for Computation and Mathematics in Electrical and Electronic Engineering, Vol. 29, No. 4, pp 964-973, 2010, https://doi.org/10.1108/03321641011044389

28. Corke, P., Wark, T., Jurdak, R., Hu, W., Valencia, P. and Moore, D., Environmental Wireless Sensor Networks, Proceedings of the IEEE, No. 11, pp 1903-1917, 2010, https://doi.org/10.1109/JPROC.2010.2068530 